\title{
Aneurisma del cayado aórtico roto. Reparación exitosa en un solo período de paro circulatorio
}

\author{
D ouglas $\mathrm{G}$ reig U ${ }^{1}$, Ricardo Zalaquett $\mathrm{S}^{1}$, Claudia Carvajal $\mathrm{F}^{2}$. \\ Complete repair in a single period \\ of circulatory arrest of a ruptured \\ atherosclerotic aortic arch aneurysm. \\ Report of one case
}

The aortic arch aneurysm is a complex disease with a growing incidence in our population. We report a 64 year-old female with an atherosclerotic aortic arch aneurysm with a maximum diameter of $9.4 \mathrm{~cm}$ and evidence of contained rupture. We performed a complete aortic arch replacement in a single period of 100 minutes of circulatory arrest with deep hypothermia and brain protection with anterograde cerebral perfusion through the right axillary artery. The patient had a complete recovery without evidence of neurological damage and was extubated on the next morning. One year later, the patient is asymptomatic and the peri-aortic haematoma was completely reabsorbed (Rev Méd Chile 2009; 137: 1346-50).

(Key words: Aneurism, ruptured; Aortic aneurism; Thoracic surgery)

Recibido el 6 de marzo, 2009. Aceptado el 25 de marzo, 2009.

${ }^{1}$ Departamento de Enfermedades Cardiovasculares. ${ }^{2}$ Departamento de Anestesiología, Facultad de Medicina, Pontificia Universidad Católica de Chile. Santiago de Chile.

$\mathrm{E}^{\prime}$ cayado aórtico es el segmento de la aorta del tronco innominado y el origen distal de la arteria subclavia izquierda. Este segmento vincula la circulación sistémica con la circulación cerebral y la circulación a las extremidades superiores, mediante los vasos supraaórticos (tronco innominado, carótida y subclavia izquierdas). Esta compleja anatomía confiere, a su vez, un complejo escenario quirúrgico, obligando a la interrupción

$\overline{\text { Correspondencia a: Dr. Ricardo Zalaquett. Marcoleta 367, }}$ 8 piso. Unidad de Cirugía Cardiovascular, Hospital Clínico Pontificia Universidad Católica de Chile. Santiago, Chile. E mail: rzalaque@med.puc.cl de la circulación sistémica y cerebral, y requiriendo, por lo tanto, procedimientos avanzados de neuroprotección ${ }^{1}$.

La incidencia anual del aneurisma de aorta torácica (AAT) es de 6/100.000 personas-año en Estados Unidos de Norteamérica y se estima que aproximadamente 30\% afectan exclusivamente a la aorta torácica y otro 30\% al segmento tóracoabdominal $^{2}$. Los aneurismas del cayado aórtico son los menos frecuentes y la rotura de éstos es una complicación catastrófica altamente letal ${ }^{3}$.

\section{Caso Clínico}

Mujer de 64 años, con antecedentes de hipertensión arterial en control médico habitual. Consultó 
en nuestro Servicio de Urgencia por dolor dorsolumbar de un mes de evolución, el cual progresó en frecuencia e intensidad en la semana previa a su ingreso, llegando a ser transfixiante y continuo en los últimos días. A su ingreso, la paciente se encontraba mal perfundida, normotensa y con taquicardia de 110 latidos por min. El examen cardiovascular, pulmonar y abdominal fue normal. La radiografía de tórax mostró un ensanchamiento del mediastino (Figura 1), por lo que se efectuó una tomografía computada, que mostró un aneurisma ateroesclerótico del arco aórtico con un diámetro máximo de 9,4 cm y evidencias de rotura contenida, con extravasación sanguínea al mediastino (Figura 2), por lo que se indicó cirugía de urgencia. La paciente fue vista en conjunto con los cirujanos vasculares, los que descartaron cualquier procedimiento endovascular.

El abordaje quirúrgico fue a través de una esternotomía media vertical. En la operación se confirmó un gran aneurisma del arco aórtico con infiltración sanguínea de toda la grasa mediastínica y cervical baja. La circulación extracorpórea y protección neurológica con perfusión cerebral anterógrada se realizó mediante la canulación de la arteria axilar derecha con interposición de un tubo protésico de dacrón de $10 \mathrm{~mm}$. Se reemplazó el arco aórtico en toda su extensión con una prótesis de dacrón de $28 \mathrm{~mm}$ durante un periodo de paro cardiocirculatorio e hipotermia profunda $\left(15^{\circ} \mathrm{C}\right)$ de 100 min de duración. Las anastomosis proximal y distal, así como la de los troncos supraaórticos, se realizaron bajo paro cardiocirculatorio, sin la interposición de clamps aórticos en ningún momento. Se mantuvo la perfusión cerebral a través de la arteria axilar con oclusión de las arterias innominada y carótida izquierda, con un tiempo total de 96 min. No se efectuó ningún tipo de monitorización neurológica. Al final del procedimiento se proporcionó perfusión cerebral retrógrada por 4 min para "purgar" el aire y eventual detrito ateroesclerótico de los vasos supraaórticos, efectuando, asimismo, maniobras de remoción de aire de las cavidades cardíacas izquierdas y de la aorta proximal, procediendo, posteriormente, a reiniciar la circulación extracorpórea y el recalentamiento sistémico.

La cirugía se completó sin incidentes y no hubo complicaciones perioperatorias. La paciente despertó a las pocas horas, sin evidencia de daño neurológico, fue extubada al primer día y dada de alta al séptimo día postoperatorio. La tomografía computada de tórax realizada un mes después mostró indemnidad del injerto, con reabsorción parcial del hematoma periaórtico (Figura 3), el cual se reabsorbió completamente en la tomografía realizada al año (no mostrada).

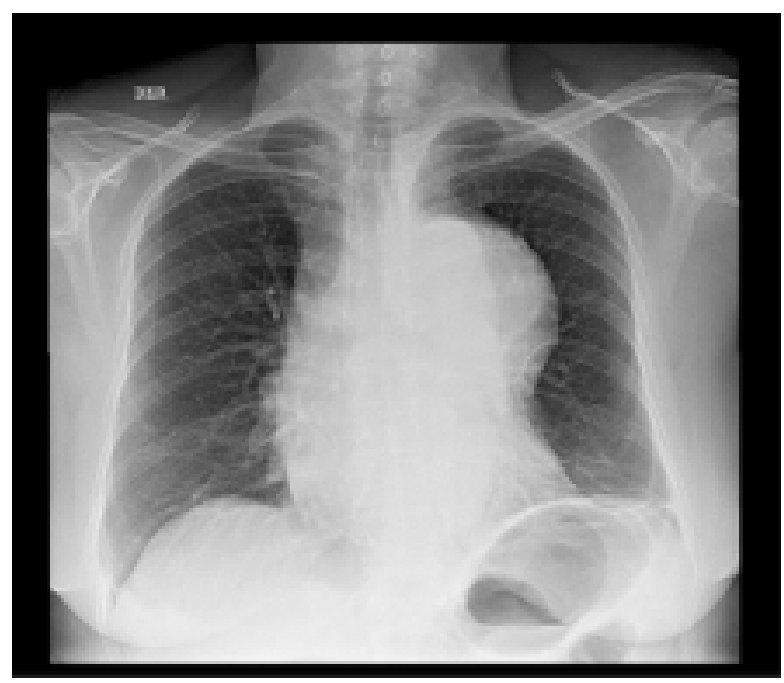

Figura 1. Radiografía de tórax al ingreso. Se observa un gran ensanchamiento mediastínico e imagen de doble contorno en relación al botón aórtico. 


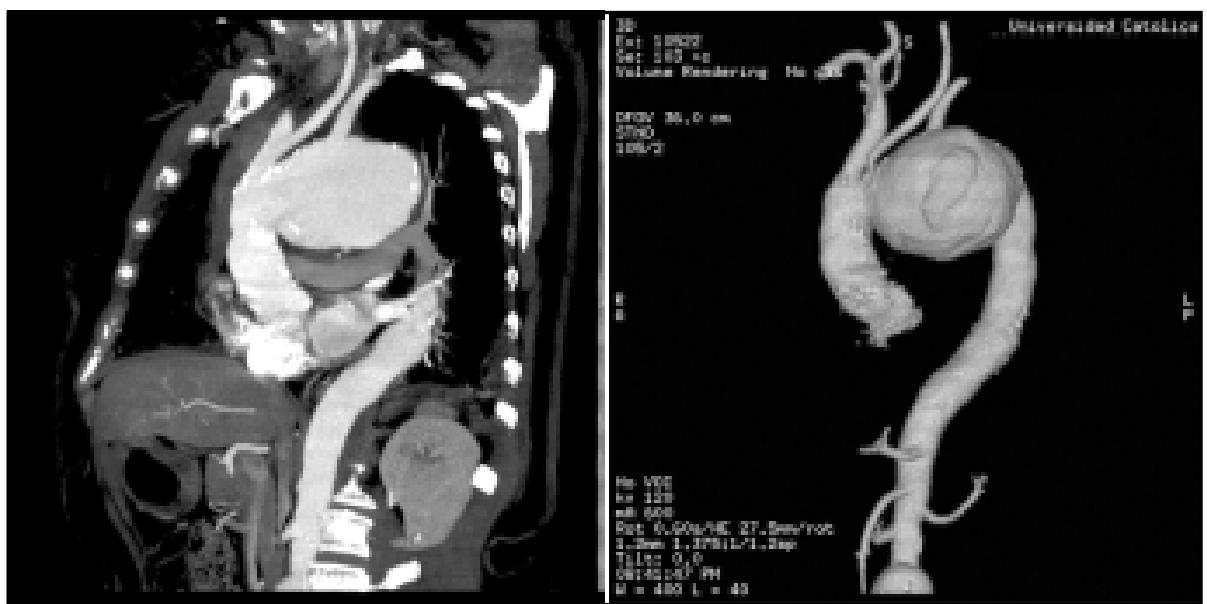

Figura 2. Tomografía computada de tórax (corte sagital y reconstrucción) que demuestra la presencia de un aneurisma de hasta 9,4 cm en el arco aórtico, con evidencias de infiltración hemática de la grasa mediastínica.

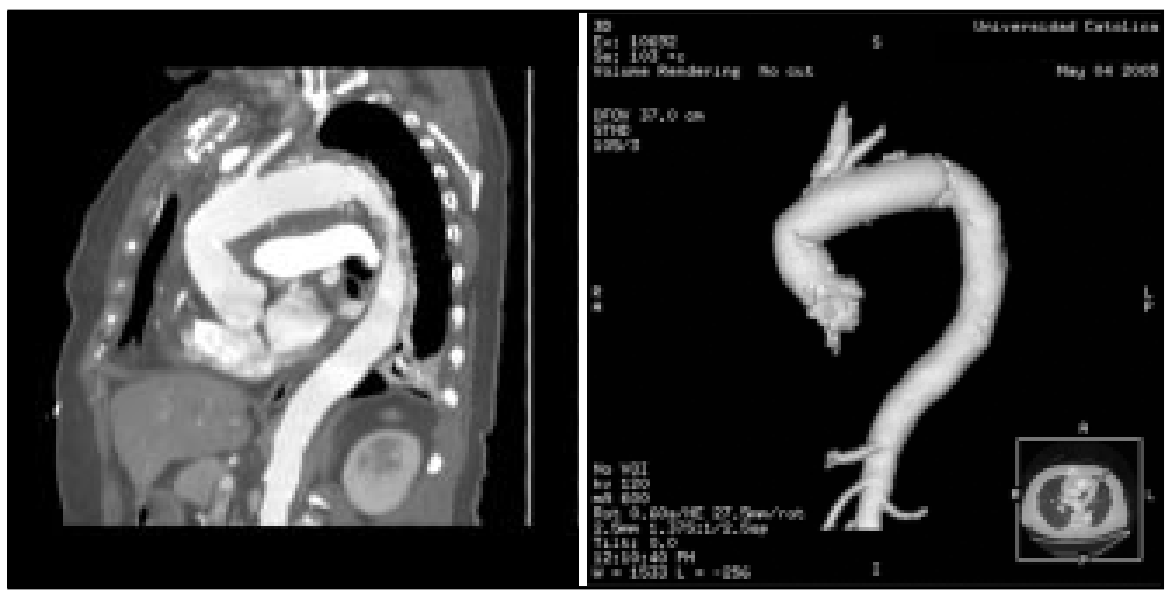

Figura 3. Tomografía computada de tórax (corte sagital y reconstrucción) que muestra el resultado postoperatorio al séptimo día (ver texto).

\section{DISCUSIÓN}

Dado el aumento de la expectativa de vida en nuestra población, con el consiguiente aumento de las comorbilidades, nos vemos enfrentados más frecuentemente a síndromes aórticos agudos, es decir, aquellos que requieren de tratamiento quirúrgico de urgencia ${ }^{3-5}$. En este caso, la paciente se presentó con evidentes signos radiológicos de extravasación sanguínea, además de intenso dolor, lo que obligó a la cirugía de urgencia.

El AAT es más frecuente en la $6^{\mathrm{a}}$ y $7^{\mathrm{a}}$ década de la vida y hasta 20\% comprometen en forma simultánea a la aorta abdominal. A diferencia del paciente joven, en que el AAT se asocia principalmente a enfermedades del tejido conectivo y al síndrome de Marfan 6 , en los pacientes mayores se asocia a la edad y a factores de riesgo cardiovasculares tales como hipertensión arterial, tabaquis- 
mo y dislipidemia. Otras causas, como válvula aórtica bicúspide, infecciosas e inflamatorias, son menos frecuentes. Histológicamente, el AAT resulta frecuentemente de la degeneración fibroquística de la capa media, lo que lleva a debilidad de la pared de la aorta ${ }^{7}$. Al respecto, reportes recientes han involucrado a la degeneración de la matriz extracelular, secundaria al aumento de la actividad de las metaloproteinasas 8 y 9 , como una de las causas del deterioro de la capa media de la aorta ${ }^{8}$.

En su mayoría, los pacientes no presentan síntomas al momento del diagnóstico. Sin embargo, al igual que en nuestra paciente, pueden presentarse con cuadros de dolor torácico, dorsal, lumbar o cuadros más graves como hemoptisis, disfagia y disnea por compresión de las estructuras vecinas. Un reporte de Davies et $\mathrm{al}^{9}$ acerca del seguimiento de 721 pacientes con aneurismas torácicos, mostró que la sobrevida a 5 años fue de $54 \%$ en los pacientes no operados y que el mejor predictor de ruptura, disección y mortalidad era el tamaño del aneurisma. De esta forma, describen que en los aneurismas mayores a $6 \mathrm{~cm}$, la ruptura ocurrió en $3,7 \%$ al año, la ruptura o disección en $6,9 \%$ al año y la muerte en $11,8 \%$ al año.

El tratamiento de la patología aórtica ha sido siempre un desafío para la cirugía. Tradicionalmente se plantean 2 accesos arteriales para la conexión a circulación extracorpórea (CEC): cuando no es posible, como en la mayoría de los casos, la canulación de la aorta ascendente: femoral y axilar (si bien se ha comunicado anecdóticamente la canulación del ápex del ventrículo izquierdo o el aneurisma mismo). Al respecto, series de pacientes no aleatorizados recomiendan esto último dado que resulta en flujo anterógrado, sin el riesgo de embolización aterosclerótica retrógrada de la canulación femoral, es más seguro en el caso de disección aórtica tipo A, evitando el flujo retrógrado por el falso lumen, y permite la perfusión cerebral anterógrada ${ }^{10,11}$, sin necesidad de canula-

\section{REFERENCIAS}

1. Kouchoukos NT, Dougenis D. Surgery of the thoracic aorta. N Engl J Med 1997; 336: 1876-88.

2. IsSELBACHER EM. Thoracic and abdominal aortic aneurysms. Circulation 2005; 111: 816-28. ción directa del tronco innominado y la carótida izquierda, lo que es engorroso, complica el campo operatorio y tiene morbilidad intrínseca.

La protección cerebral durante el paro circulatorio ha sido ampliamente estudiada. Se reconocen al menos 3 mecanismos de neuroprotección: hipotermia sistémica profunda exclusiva, perfusión cerebral retrógrada asociada (con irrigación "retrógrada" a través de vena cava superior) ${ }^{12}$ y perfusión cerebral anterógrada selectiva (con canulación de la arteria axilar o con perfusión directa por canulación de ambas carótidas) ${ }^{12-14}$. Actualmente, se reconoce a la hipotermia profunda en conjunto con la perfusión anterógrada como el estándar de neuroprotección, ya que ésta, a diferencia de la perfusión retrógrada, permite mayores tiempos de paro cardiocirculatorio y una mayor irrigación del territorio cerebral ${ }^{13,15,16}$. Por otro lado, un reporte de Urbansky et al mostró que la evaluación preoperatoria de la anatomía del polígono de Willis con angioTAC cerebral no se correlacionó con los test funcionales ni la perfusión cerebral, en pacientes sometidos a perfusión cerebral unilateral durante cirugía del arco aórtico ${ }^{17}$. De aquí que en la actualidad la perfusión de la arteria axilar derecha con oclusión proximal del tronco innominado, como ya señalamos, sea el procedimiento de elección por sobre la canulación directa de ambas carótidas comunes.

Nuestra paciente fue sometida a paro circulatorio con hipotermia sistémica profunda y perfusión cerebral anterógrada a través de la arteria axilar derecha con oclusión del tronco innominado, con un tiempo de paro circulatorio de 100 min. El resultado neurológico en el postoperatorio inmediato y alejado fue excelente, lo que es concordante con lo publicado en la literatura en este tipo de pacientes operados con los procedimientos de paro circulatorio y protección cerebral utilizados en el caso clínico que presentamos ${ }^{3,18}$.

3. IrarráZaval Ll MJ, Morán VS, Zalaquett SR, Becker RP, BaEza PC, URzúa UJ et al. [Partial or total replacement of the aortic arch. Experience in 23 patients]. Rev Méd Chile 2006; 134: 575-80.

4. Ueda Y. [Recent advances in thoracic aortic surgery]. Nippon Geka Gakkai Zasshi 2008; 109: 201-4. 
5. InCE H, NiEnABer CA. Etiology, pathogenesis and management of thoracic aortic aneurysm. Nat Clin Pract Cardiovasc Med 2007; 4: 418-27.

6. Judge DP, Dietz HC. Marfan's syndrome. Lancet 2005; 366: 1965-76

7. Galis ZS, Khatri JJ. Matrix metalloproteinases in vascular remodeling and atherogenesis: the good, the bad, and the ugly. Circ Res 2002; 90: 251-62.

8. Wilson WR, Anderton M, Schwalbe EC, Jones JL, Furness PN, Bell PR ET AL. Matrix metalloproteinase-8 and -9 are increased at the site of abdominal aortic aneurysm rupture. Circulation 2006; 113: 438-45.

9. Davies RR, Goldstein LJ, Coady MA, Tittle SL, Rizzo JA, Kopf GS ET AL. Yearly rupture or dissection rates for thoracic aortic aneurysms: simple prediction based on size. Ann Thorac Surg 2002; 73: 17-27.

10. Gulbins H, Pritisanac A, Ennker J. Axillary versus femoral cannulation for aortic surgery: enough evidence for a general recommendation? Ann Thorac Surg 2007; 83: 1219-24.

11. Zalaquett R, Bahamondes JC, Mertens R, Morán S, Irarrázaval MJ, Becker P et Al. [Axillary artery cannulation for extracorporeal circulation]. Rev Méd Chile 2003; 131: 390-6.

12. Zalaquett R, irarrázaval MJ, Morán S, Muñoz C, Garayar B, Becker P et al. [Retrograde cerebral perfusion during circulatory arrest with deep hypothermia. A new technique for brain protection in surgery of ascending aorta and aortic arch]. Rev Méd Chile 1995; 123: 1489-98.

13. Apostolakis E, Shuhaiber JH. Antegrade or retrograde cerebral perfusion as an adjunct during hypothermic circulatory arrest for aortic arch surgery. Expert Rev Cardiovasc Ther 2007; 5: 1147-61.

14. Dias RR, Silva IA, Fiorelli AI, Stolf NA. Cerebral protection: sites of arterial cannulation and brain perfusion routes. Rev Bras Cir Cardiovasc 2007; 22: 235-40.

15. Malvindi PG, Scrascia G, Vitale N. Is unilateral antegrade cerebral perfusion equivalent to bilateral cerebral perfusion for patients undergoing aortic arch surgery? Interact Cardiovasc Thorac Surg 2008; 7: 891-7.

16. Kawata M, ТАкамото S. [Protection of the central nervous system during thoracic aortic surgery]. Masui 2007; 56: 271-9.

17. Urbanski PP, Lenos A, Blume JC, Ziegler V, Griewing B, Schmitt R ET AL. Does anatomical completeness of the circle of Willis correlate with sufficient cross-perfusion during unilateral cerebral perfusion? Eur J Cardiothorac Surg 2008; 33: 402-8.

18. Yagdi T, Atay Y, Cikirikcioglu M, Boga M, Posacioglu H, Ozbaran M et al. Determinants of early mortality and neurological morbidity in aortic operations performed under circulatory arrest. J Card Surg 2000; 15: 186-93. 